\title{
On the Resolvent of a Linear Operator Associated with a Well-Posed Cauchy Problem
}

\author{
By John Miller
}

Summary. We show how local estimates may be obtained for holomorphic functions of a class of linear operators on a finite-dimensional linear vector space. This is accomplished by classifying the spectrum of each operator and then estimating its resolvent on certain contours in the left half-plane. We apply these methods to prove some known theorems, and in addition we obtain new estimates for the inverse of these operators. Analogous results for power-bounded operators are given in [3].

1. Introduction. Let $\mho_{m}$ be $m$-dimensional unitary space and $J$ the family of all endomorphisms of $\mho_{m}$. Let $(u, v)$ denote the scalar product of elements of $V_{m}$, then on $V_{m}$ the vector norm is defined by $|v|=(v, v)^{1 / 2}$ and the operator norm by $|T|$ $=\sup _{|v|=1}|T v|$. We are interested in the subset $\Im(C)$ of operators in $\Im$ which satisfy, for each $v \in \mathcal{V}_{m}$ and some constant $C(v)$ depending only on $v$, the local resolvent condition

$$
\left|(z I-T)^{-1} v\right| \leqq C(v) / \operatorname{Re} z
$$

for all complex numbers $z$ in the open right half-plane. For example, the global resolvent condition

$$
\left|(z I-T)^{-1}\right| \leqq C / \operatorname{Re} z
$$

for all complex numbers $z$ in the open right half-plane implies (1.1) at each point $v \in v_{m}$ with $C(v)=C \cdot|v|$.

Remark 1.1. $\Im(C)$ contains operators of practical importance because if $u(x, t)$ is a solution of the well-posed Cauchy problem

$$
\begin{aligned}
& \frac{\partial u(x, t)}{\partial t}=T u(x, t), \quad u(x, 0)=f(x), \quad|u(x, t)| \leqq C|f(x)|, \\
& -\infty<x<\infty, \quad t>0
\end{aligned}
$$

where $T$ is a matrix of complex numbers, then $u(x, t)=e^{T t} u(x, 0)$ where for some constant $C^{\prime}$

$$
\left|e^{T t}\right| \leqq C^{\prime}
$$

for all $t \geqq 0$. It is a standard result, easily seen by taking the Laplace transform of $e^{T t}$, that (1.3) implies condition (1.2).

For each $T \in J(C)$ we consider the family of functions $\mathcal{F}(T)$ which are holomorphic in a sufficiently large open neighbourhood of $\sigma(T)$, the spectrum of $T$.

Remark 1.2. It is easy to check that condition (1.1) implies that, for each $T$ $\in \Im(C), \sigma(T)$ is contained in the closed left half-plane, and that points of $\sigma_{0}(T)$ (i.e.,

Received September 18, 1967. 
those points of $\sigma(T)$ on the imaginary axis) are simple poles of the resolvent function $(z I-T)^{-1}$.

In what follows for each $T \in J(C)$ we classify the points of $\sigma(T)$ not in $\sigma_{0}(T)$, and then for each class we construct simple closed rectifiable Jordan curves which decompose the points of the class into disjoint subsets.

Remark 1.3. It will be seen later that $\mathcal{F}(T)$ can be defined to be those functions which are holomorphic on any open neighbourhood of $\sigma_{0}(T)$ and the compact sets bounded by the curves associated with the above subsets of eigenvalues. In particular any function holomorphic in an open neighbourhood of the closed left half-plane is in $\mathcal{F}(T)$ for every $T \in \Im(C)$.

2. Classification of the Spectrum. For each $T \in J$ we now classify the points of $\sigma(T)$ not in $\sigma_{0}(T)$ in terms of their distance from the imaginary axis. We start by drawing a line parallel to the imaginary axis through each of these points. These lines together with the imaginary axis form a set $\&$ of lines lying in the closed left half-plane. Each pair of adjacent lines in $\&$ bounds a doubly infinite open strip containing no elements of $\sigma(T)$. The set of these open strips with the order relation "the strip $s$ ' follows the strip $s$ if $s^{\prime}$ is to the left of $s$ " forms a sequence $\delta$. We are interested in the subsequence $\delta_{1}$ of $\delta$ constructed as follows. Its first strip is the same as that of $\delta$, namely the strip bounded by the imaginary axis and the first element of $\&$ to the left of it. Let $w_{\alpha}(T)$ and $\delta_{\alpha}(T)$ denote respectively the width of the $\alpha$ th strip of $\delta_{1}$ and the distance of its left boundary from the imaginary axis. Then the $\alpha$ th strip $(\alpha>1)$ of $\delta_{1}$ is defined to be the first strip of $\delta$ following the $(\alpha-1)$ th of $\delta_{1}$ whose width is greater than $\delta_{\alpha-1}(T)$. We have therefore

$$
w_{1}(T)=\delta_{1}(T)
$$

and

$$
w_{\alpha}(T)>\delta_{\alpha-1}(T)
$$

for $\alpha>1$.

We now prove that

$$
\delta_{\alpha}(T)>w_{\alpha}(T)>\delta_{\alpha}(T) / m
$$

for $\alpha>1$. The left inequality is immediate from the definitions. The right follows by observing that $\delta_{\alpha}(T)$ is the sum of $w_{\alpha}(T)$, and the distance between the right boundary of the $\alpha$ th strip of $\delta_{1}$ and the left boundary of the $(\alpha-1)$ th strip of $\delta_{1}$, and $\delta_{\alpha-1}(T)$. From the construction of the $\alpha$ th strip, and the fact that there are at most $m$ strips in $\delta$, it follows that the middle quantity is at most $(m-2) \delta_{\alpha-1}(T)$. Using (2.2) we now see that $\delta_{\alpha}(T)<m w_{\alpha}(T)$ as required.

For $\alpha \geqq 1$ we define the $\alpha$ th class $\sigma_{\alpha}(T)$ to be those points in $\sigma(T)$ lying between the $\alpha$ th and $(\alpha+1)$ th strips of $S_{1}$.

Remark 2.1. The points in $\sigma_{\alpha}(T)$ nearest to the imaginary axis are a distance $\delta_{\alpha}(T)$ from it, and no point in $\sigma_{\alpha}(T)$ is farther than $m \delta_{\alpha}(T)$ from it. Inequalities (2.2) and (2.3) imply that the distance of $\sigma_{\alpha}(T)$ from any other class is greater than $\delta_{\alpha}(T) / m$.

3. The Spectral Decomposition. We now decompose the spectrum $\sigma\left(T^{\prime}\right)$, for 
each operator $T \in J(C)$, into disjoint spectral sets. Each distinct eigenvalue in $\sigma_{0}(T)$ forms a distinct spectral set. For each such spectral set we draw a circle with its element as centre and arbitrarily small radius, certainly less than half the distance between its centre and the rest of the spectrum. We orientate each such circle in the usual way, so that its centre is to the left of the circle.

To construct the spectral sets within the general class $\sigma_{\alpha}(T)$ we proceed as follows. About each point in the class we draw a circle with the point as centre and radius $r_{\alpha}(T)$, where

$$
r_{\alpha}(T)=r \delta_{\alpha}(T) / 2 m
$$

and $r$ is any fixed number in the interval $(0,1]$. (3.1) and Remark 2.1 ensure that circles of different classes do not intersect. If two circles about points in $\sigma_{\alpha}(T)$ intersect, we combine them into a single contour by deleting any part of a circle lying in the interior of another. Proceeding in this manner we obtain a finite number of disjoint closed rectifiable Jordan curves, which we orientate in the usual manner. Taken together these surround all the points of $\sigma_{\alpha}(T)$, and the eigenvalues enclosed by any one of these are taken to be a spectral set of this class.

Taking the spectral sets in $\sigma_{0}(T)$ and those to the left of the imaginary axis, we obtain a decomposition of $\sigma(T)$ into a finite number of disjoint components, of which the associated contours form an orientated envelope of $\sigma(T)$ denoted by $\gamma(r, T)$.

Remark 3.1. The length of any component of $\gamma(r, T)$ in $\sigma_{\alpha}(T)$ is at most $2 \pi m r_{\alpha}(T)$. The distance between any two points in its associated spectral set is not more than $2(m-1) r_{\alpha}(T)$. Remark 2.1 and (3.1) imply that the distance of any point on such a component from $\sigma(T)$ is at least $r_{\alpha}(T)$.

In what follows we denote by $C_{l}(T)$ an arbitrary spectral set in the above decomposition of $\sigma(T)$, and we let $\gamma_{l}(r, T)$ denote the associated component of $\gamma(r, T)$. It is clear that $\sigma(T)=\bigcup_{\alpha} \sigma_{\alpha}(T)=\bigcup_{l} C_{l}(T)$ and $\gamma(r, T)=\bigcup_{l} \gamma_{l}(r, T)$. Corresponding to each $C_{l}(T)$ we define the operator

$$
E_{l}(T)=\frac{1}{2 \pi i} \oint_{\gamma_{l}(r, T)}(z I-T)^{-1} d z
$$

then it is well known that

$$
E_{k}(T) E_{l}(T)=\delta_{k l} E_{l}(T), \quad \sum_{l} E_{l}(T)=I, \quad E_{l}(T) T=T E_{l}(T) .
$$

Also for any $v \in \mathcal{V}_{m}, T \in J(C)$ and $f \in \mathcal{F}(T)$ we have

$$
f(T) v=\frac{1}{2 \pi i} \oint_{\gamma(r, T)} f(z)(z I-T)^{-1} v d z
$$

and

$$
E_{l}(T) f(T) v=\frac{1}{2 \pi i} \oint_{\gamma_{l}(r, T)} f(z)(z I-T)^{-1} v d z .
$$

From Remark 1.2 it follows that if $C_{l}(T)=\left\{\kappa_{l}(T)\right\}$, where $\kappa_{l}(T) \in \sigma_{0}(T)$, we obtain in this case an alternate expression to (3.5), namely

$$
E_{l}(T) f(T) v=f\left(\kappa_{l}(T)\right) \lim _{z \rightarrow \kappa_{l}(T)}\left(z-\kappa_{l}(T)\right)(z I-T)^{-1} v
$$


Using (3.6) for the spectral sets in $\sigma_{0}(T)$ and (3.5) for the others we now see the truth of Remark 1.3.

In the applications presented below the functions defined in Remark 1.3 are sufficient. However it may arise in other situations that the functions have singularities closer to the spectrum of one or more of the operators in J. In such a case it is necessary to construct a smaller orientated envelope than the one given above. This is done in detail in [3] for a particular case, and analogous methods are applicable here, although we do not pause to develop them.

4. Estimation of the Resolvent. The resolvent of any operator $T \in J(C)$ satisfies (1.1) in the open right half-plane. This operator-valued function of $z$ is holomorphic in the resolvent set and it is important to have an estimate of it throughout this set. To obtain such an estimate we consider the polynomial in $z$ of degree at most $m-1$ given by

$$
P(z ; T)=(z I-T)^{-1} \prod_{j=1}^{m}\left(z-\kappa_{j}(T)\right)
$$

where $\sigma(T)=\left\{\kappa_{1}(T), \cdots, \kappa_{m}(T)\right\}$. If $Z$ is any point in the complex plane we can represent $P(z ; T)$ by a Cauchy-Taylor series about $Z$. Thus

$$
P(z ; T)=\sum_{k=0}^{m-1}(z-Z)^{k} \frac{1}{2 \pi i} \oint_{\Gamma} \frac{P(s ; T)}{(s-Z)^{k+1}} d s
$$

where $\Gamma$ is any closed contour surrounding $Z$. Combining (4.1) and (4.2) we see that for any point $z$ in the resolvent set of $T$ we can write

$$
(z I-T)^{-1}=\frac{1}{2 \pi i} \oint_{\Gamma} \varphi(s, z ; T)(s I-T)^{-1} d s
$$

where

$$
\varphi(s, z ; T)=\frac{1}{s-Z} \prod_{j=1}^{m}\left(\frac{s-\kappa_{j}(T)}{z-\kappa_{j}(T)}\right) \sum_{k=0}^{m-1}\left(\frac{z-Z}{s-Z}\right)^{k} .
$$

From (1.1) and (4.3) we see that for any $v \in \mathcal{V}_{m}, T \in I(C)$ and $z$ in the resolvent set of $T$

$$
\left|(z I-T)^{-1} v\right|<\frac{|\Gamma|}{2 \pi} C(v) \sup _{s}\left|\frac{\varphi(s, z ; T)}{\operatorname{Re} s}\right|, \quad(s \in \Gamma)
$$

where $|\Gamma|$ denotes the length of $\Gamma$.

In particular, to estimate the resolvent on the component $\gamma_{l}(r, T)$ associated with a typical spectral set $C_{l}(T)$ in $\sigma_{\alpha}(T)$ we choose $Z=Z_{l}(T)$ to be the point with real part $2 \delta_{\alpha}(T)$ and imaginary part equal to the imaginary part of $\kappa_{l}(T)$, where $\kappa_{l}(T)$ is a point in $C_{l}(T)$ nearest to the imaginary axis. The corresponding contour $\Gamma=\Gamma_{l}(T)$ is taken to be the circle with centre $Z_{l}(T)$ and radius $\delta_{\alpha}(T)$. Then it is clear that $\left|\Gamma_{l}(T)\right|=2 \pi \delta_{\alpha}(T)$ and $|\operatorname{Re} s| \geqq \delta_{\alpha}(T)\left(s \in \Gamma_{l}(T)\right)$, so that (4.5) implies for any $z \in \gamma_{l}(r, T)$ and $v \in \mathcal{V}_{m}$

$$
\left|(z I-T)^{-1} v\right| \leqq C(v) \sup _{s}|\varphi(s, z ; T)|, \quad\left(s \in \Gamma_{l}(T)\right) .
$$


Also for $s \in \Gamma_{l}(T), \kappa_{j} \in \sigma(T)$ and $z \in \gamma_{l}(r, T)$ we have

$$
\left|s-Z_{\iota}(T)\right|=\delta_{\alpha}(T)
$$

and from Remarks 2.1 and 3.1 it is easy to verify that $\left|z-\kappa_{j}(T)\right| \geqq r_{\alpha}(T)$, $\left|z-Z_{l}(T)\right| \leqq(m+2) \delta_{\alpha}(T)+r_{\alpha}(T),|z-s| \leqq(m+3) \delta_{\alpha}(T)+r_{\alpha}(T)$. Thus, from (3.1) and the fact that

$$
\frac{s-\kappa_{j}(T)}{z-\kappa_{j}(T)}=1-\frac{z-s}{z-\kappa_{j}(T)},
$$

we obtain for any $s \in \Gamma_{l}(T), \kappa_{j} \in \sigma(T)$ and $z \in \gamma_{l}(r, T)$

$$
\left|\frac{z-Z_{l}(T)}{s-Z_{l}(T)}\right|<m+3, \quad\left|\frac{s-\kappa_{j}(T)}{z-\kappa_{j}(T)}\right|<\frac{2 m(m+4)}{r} .
$$

Combining (4.4), (4.7) and (4.8) we see that for any $s \in \Gamma_{l}(T)$ and $z \in \gamma_{l}(r, T)$

$$
|\varphi(s, z ; T)| \leqq \frac{k(m)}{r^{m-1} r_{\alpha}(T)}
$$

where

$$
k(m)=m(m+4)^{m}(2 m(m+3))^{m-1}<e^{7 m^{2}} .
$$

Therefore from (4.6) and (4.9) we have for any $v \in \mathcal{V}_{m}$ and $z \in \gamma_{l}(r, T)$

$$
\left|(z I-T)^{-1} v\right| \leqq \frac{k(m) C(v)}{r^{m-1} r_{\alpha}(T)} .
$$

Recalling Remark 3.1 and putting dist $(z, \sigma(T))=\min _{j}\left|z-\kappa_{j}(T)\right|$, the estimate (4.11) establishes the following.

THEOREM 4.1. For any $T \in J(C)$ there exists an orientated envelope $\gamma(r, T)$ of $\sigma(T)$ such that for any $v \in \mathcal{V}_{m}$ and $z \in \gamma(r, T)$

$$
\left|(z I-T)^{-1} v\right| \leqq \frac{k(m) C(v)}{r^{m-1} \operatorname{dist}(z, \sigma(T))}
$$

where $k(m)<e^{7 m^{2}}$ and $r$ is any number in the interval $(0,1]$.

Using the maximum modulus principle and choosing $r$ sufficiently small it is clear that Theorem 4.1 provides a local estimate of the resolvent function at any point in the resolvent set.

5. The Basic Lemmas. We now state and prove two basic lemmas, which follow easily from the results of the previous section.

Lemma 5.1. If $v \in \mathcal{V}_{m}, T \in J(C)$ and $E_{l}(T)$ is the projection associated with a spectral set $\left\{\kappa_{l}(T)\right\}$ in $\sigma_{0}(T)$, then for any $f \in \mathcal{F}(T)$ we have

$$
\left|E_{l}(T) f(T) v\right| \leqq\left|f\left(\kappa_{l}(T)\right)\right| C(v) .
$$

Proof. Put $z(\epsilon)=\kappa_{l}(T)+\epsilon, \epsilon>0$, in expression (3.6) and observe that $z(\epsilon)-\kappa_{l}(T)=\epsilon$ and $\operatorname{Re} z(\epsilon)=\epsilon$. Then using (1.1) it follows that

$$
\left|\left(z(\epsilon)-\kappa_{l}(T)\right)(z(\epsilon) I-T)^{-1} v\right| \leqq C(v)
$$


for each $\epsilon>0$, and therefore $\left|E_{l}(T) f(T) v\right| \leqq\left|f\left(\kappa_{l}(T)\right)\right| C(v)$. Q.E.D.

Lemma 5.2. If $v \in \mathcal{V}_{m}, T \in I(C)$ and $E_{l}(T)$ is the projection associated with a spectral set $C_{l}(T)$ in $\sigma_{\alpha}(T)(\alpha \geqq 1)$, then for any $f \in \mathcal{F}(T)$ we have

$$
\left|E_{l}(T) f(T) v\right| \leqq \frac{k_{1}(m) C(v)}{r^{m-1}} \sup _{z}|f(z)|, \quad\left(z \in \gamma_{l}(r, T)\right),
$$

where $k_{1}(m)<e^{8 m^{2}}$ and $r$ is any number in the interval $(0,1]$.

Proof. From Eq. (3.5) we have

$$
\left|E_{l}(T) f(T) v\right| \leqq \frac{\left|\gamma_{l}(r, T)\right|}{2 \pi} \sup _{z}\left|f(z)(z I-T)^{-1} v\right|, \quad\left(z \in \gamma_{l}(r, T)\right)
$$

which, together with (4.11) and Remark 3.1, implies the lemma with $k_{1}(m)=$ $m k(m)$. Q.E.D.

Combining Lemmas 5.1 and 5.2 and observing that $f(T)=\sum_{l} E_{l}(T) f(T)$ we obtain the following theorem, which gives a local estimate for a function of an operator. For convenience we let $\gamma^{\prime}(r, T)$ denote those components of $\gamma(r, T)$ associated with spectral sets not in $\sigma_{0}(T)$.

Theorem 5.3. If $v \in \mathcal{V}_{m}$ and $T \in \mathfrak{I}(C)$ then for any $f \in \mathcal{F}(T)$ we have

$$
|f(T) v| \leqq \frac{K(m) C(v)}{r^{m-1}} \sup _{z}|f(z)|, \quad\left(z \in \sigma_{0}(T) \cup \gamma^{\prime}(r, T)\right),
$$

where $K(m)<e^{9 m^{2}}$ and $r$ is any number in the interval $(0,1]$.

6. Applications. In this section we derive several results which are a consequence of Lemmas 5.1 and 5.2. The first of these is a theorem which includes the exponential analogue of a result proved by Morton in [4]. Its novelty, however, is that it also provides an estimate not only for positive values but also for negative values of the exponential variable $t$. For convenience we put $\tau(T)=\max _{j} \operatorname{Re} \kappa_{j}(T)$ and $\mu(T)=$ $\min _{j} \operatorname{Re} \kappa_{j}(T)$.

THeorem 6.1. If $v \in \mathcal{V}_{m}$ then for any $T \in I(C)$ we have

$$
\left|e^{T t} v\right| \leqq \frac{\exp [(1-r / 2 m) \tau(T) t] K(m) C(v)}{r^{m-1}}
$$

for all $t \geqq 0$, and

$$
\left|e^{T t} v\right| \leqq \frac{\exp [(1+r / 2 m) \mu(T) t] K(m) C(v)}{r^{m-1}}
$$

for all $t<0$, where $K(m)<e^{9 m^{2}}$ and $r$ is any number in the interval $(0,1]$.

Proof. To prove this we observe that for each fixed $t$ the function $f_{t}(z)=e^{z t}$ is entire and hence it is in $\mathcal{F}(T)$ for each $T \in J(C)$. We construct $\gamma(r, T)$ for each $T$ $\in \Im(C)$. Clearly if $z \in \sigma_{0}(T)$ then $\left|e^{z t}\right|=1$, and if $z \in \gamma^{\prime}(r, T)$ we have $(1+r / 2 m) \mu(T)$ $\leqq \operatorname{Re} z \leqq(1-r / 2 m) \tau(T)$. Therefore for $z \in \sigma_{0}(T) \cup \gamma^{\prime}(r, T)$

$$
\left|e^{z t}\right| \leqq \exp [(1-r / 2 m) \tau(T) t]
$$

for $t>0$, and 


$$
\left|e^{2 t}\right| \leqq \exp [(1+r / 2 m) \mu(T) t]
$$

for $t<0$. Using (6.1) and (6.2) in Theorem 5.3 we obtain the first and second parts respectively of the theorem. Q.E.D.

Our second application is to the proof of a sharp form of a theorem of Kreiss [1]. This sharp form was first given in [2] by an indirect method.

THeorem 6.2. If $v \in \mathcal{V}_{m}$, then for each $T \in J(C)$ there exists a positive-definite Hermitean operator $H(T)$ such that $H(T) T+T^{*} H(T) \leqq 2(1-r) \tau(T) H(T)$ with $H(T) \geqq I$ and $(v, H(T) v)^{1 / 2} \leqq r^{-m+1} K(m) C(v)$, where $K(m)<e^{9 m^{2}}$ and $r$ is any number in the interval $(0,1]$.

Proof. For each $T \in J(C)$ we construct the orientated envelope $\gamma(r, T)$. With $E_{l}(T)$ defined by (3.2), we put $T_{l}=E_{l}(T) T$ and we construct below an $H_{l}(T)$ for each spectral set $C_{l}(T) . H(T)$ is then defined as

$$
H(T)=m \sum_{l} H_{l}(T) .
$$

If $\left\{\kappa_{l}(T)\right\}$ is a spectral set in $\sigma_{0}(T)$ we put $H_{l}(T)=E_{l}{ }^{*}(T) E_{l}(T)$. But $T_{l}=$ $\kappa_{l}(T) E_{l}(T)$ and therefore

$$
H_{l}(T) T_{l}+T_{l}{ }^{*} H_{l}(T)=2 \operatorname{Re} \kappa_{l}(T) E_{l}{ }^{*}(T) E_{l}(T)=0 .
$$

Also, by Lemma 5.1 we have $\left|E_{l}(T) v\right| \leqq C(v)$ so that

$$
\left(v, H_{l}(T) v\right)^{1 / 2} \leqq C(v) .
$$

On the other hand if $C_{l}(T)$ is a spectral set in $\sigma_{\alpha}(T)(\alpha \geqq 1)$ and $\kappa_{l}(T)$ is a point in $C_{l}(T)$ nearest to the imaginary axis, Remark 3.1 implies that $\left|z-\kappa_{l}(T)\right| \leqq$ $(2 m-1) r_{\alpha}(T)$ for any $z \in \gamma_{l}(r, T)$.

Therefore if $g_{l}{ }^{n}(z)=\left(\left(z-\kappa_{l}(T)\right) / r \delta_{\alpha}(T)\right)^{n}$ and

$$
g_{l}{ }^{n}(T)=\frac{1}{2 \pi i} \oint_{\gamma_{l}(r, T)} g_{l}{ }^{n}(z)(z I-T)^{-1} d z
$$

we have $\left|g_{l}{ }^{n}(z)\right| \leqq(1-1 / 2 m)^{n}$ for all $z \in \gamma_{l}(r, T)$, and Lemma 5.2 implies that

$$
\left|g_{l}{ }^{n}(T) v\right| \leqq\left(1-\frac{1}{2 m}\right)^{n} \frac{k_{1}(m) C(v)}{r^{m-1}}
$$

where $k_{1}(m)<e^{8 m^{2}}$.

We put $H_{l}(T)=\sum_{n=0}^{\infty}\left(g_{l}{ }^{n}(T)\right)^{*} g_{l}{ }^{n}(T)$ and therefore

$$
\left(v, H_{l}(T) v\right)^{1 / 2} \leqq\left(\frac{4 m}{3}\right)^{1 / 2} \frac{k_{1}(m) C(v)}{r^{m-1}} .
$$

Now $T_{l}=\kappa_{l}(T) E_{l}(T)+r \delta_{\alpha}(T) g_{l}(T)$ so that $H_{l}(T) T_{l}+T_{l}{ }^{*} H_{l}(T)=$ $2 r \operatorname{Re} \kappa_{l}(T) H_{l}(T)+r \delta_{\alpha}(T) G_{l}(T)$ where $G_{l}(T)=H_{l}(T) g_{l}(T)+g_{l}{ }^{*}(T) H_{l}(T)$. But for all nonnegative integers $n$

$$
\begin{aligned}
&\left(g_{l}{ }^{n+1}(T)\right)^{*} g_{l}{ }^{n}(T)+\left(g_{l}{ }^{n}(T)\right)^{*} g_{l}{ }^{n+1}(T) \\
& \leqq\left(g_{l}{ }^{n+1}(T)\right)^{*} g_{l}{ }^{n+1}(T)+\left(g_{l}{ }^{n}(T)\right)^{*} g_{l}{ }^{n}(T) .
\end{aligned}
$$

Summing (6.7) from $n=0$ to $\infty$ we see that $G_{l}(T) \leqq 2 H_{l}(T)$ and therefore

$$
H_{l}(T) T_{l}+T_{l}^{*} H_{l}(T) \leqq 2 \tau(T)(1-r) H_{l}(T) .
$$


The expressions (6.4) and (6.8) taken together verify the first property required of $H(T)$. The last property follows from $(6.3),(6.5)$ and (6.6). It remains to show that $H(T) \geqq I$, but $(v, v)=\left|\sum_{l} E_{l}(T) v\right|^{2} \leqq m \sum_{l}\left|E_{l}(T) v\right|^{2} \leqq(v, H(T) v)$. Q.E.D.

It would be interesting to know if, for certain classes of linear operators on Hilbert space satisfying (1.1), it is possible to construct orientated envelopes of their spectra on which Lemmas 5.1 and 5.2 hold. The best estimate for $K(m)$ in Theorem 5.3 is also unknown.

University of Massachusetts

Boston, Massachusetts 02116

1. H.-O. KREISs, "Über Matrizen die beschränkte Halbgruppen erzeugen," Math. Scand., v. 7, 1959, pp. 71-80.

2. John Miller \& Gilbert Strang, "Matrix theorems for partial differential and difference equations," Math. Scand., v. 18, 1966, pp. 113-133. MR 35 \#206.

3. JOHN Miller, "On power-bounded operators and operators satisfying a resolvent condition," Numer. Math., v. 10, 1967, pp. 389-396.

4. K. W. Morton, "On a matrix theorem due to H.-O. Kreiss," Comm. Pure Appl. Math., v. 17,1965 , pp. $375-380$. MR $30 \# 698$. 